\title{
Smart structures and intelligent systems for health monitoring and diagnostics
}

doi:10.1533/abbi.2004.0015

\author{
M. A. El-Sherif \\ Department of Materials and Electrical and Computer Engineering, Fiber Optics and Photonics, \\ Manufacturing Engineering Center, Drexel University, Philadelphia, PA 19104, USA
}

\begin{abstract}
Smart and intelligent" structures are defined as structures capable of monitoring their own "health" condition and structural behavior, such structures are capable of sensing external environmental conditions, making decisions, and sending the information to other locations. Available conventional devices and systems are not technologically mature for such applications. New classes of miniature devices and networking systems are urgently needed for such applications. In this paper, two examples of the successful work achieved so far, in biomedical application of smart structures, are presented. The first one is based on the development of a smart bone fixation device for rehabilitation and treatment. This device includes a smart composite bar that can sense physical stress applied to the fractured bones, and send the information to the patient's physician remotely. The second is on the development of smart fabrics for many applications including health monitoring and diagnostics. Successful development of such smart fabrics with embedded fiber optic sensors and networks is mainly dependent on the development of the proper miniature sensor technology, and on the integration of these sensors into textile structures. The developed smart structures will be discussed and samples of the results will be presented.
\end{abstract}

Key words: Smart structures, bone fixation devices, smart fabrics/textiles, electronic fabrics/textiles, biomedical systems, fiber optic sensors, remote sensors.

\section{INTRODUCTION}

During the twentieth century, tremendous progress has been achieved in the field of biomedical engineering. This achievement can be attributed to the development of novel electronics and optical devices and systems. Integrated circuits and fiber optics developed and processed in microstructural devices were behind marvelous achievements in biomedical engineering. A portable blood analysis device, the size of a matchbox, is an example of how crucial integrated circuits and fiber optics are.

In the twenty-first century, the development of smart structures and intelligent systems will form the basis for more innovation in biomedical engineering applications. Ranging from health monitoring and diagnostic systems to intelligent artificial body parts, such as the Smart Artificial Heart. The smart artificial hearts will be able to

Correspondence Author:

Prof M. A. El-Sherif

Department of Materials and Electrical \& Computer Engineering

Fiber Optics and Photonics, Manufacturing Engineering Center

Drexel University, Philadelphia, PA 19104, USA

Tel: 215 387-9970 Fax: 215 387-4520

Email: melsherif@photonicslabs.com accommodate any physical changes in the body and modify the blood circulation to optimize the health conditions. Health monitoring shirts for special care will have direct communication with the patient's physician and will feature feedback control of a through-the-skin drug delivery system. A clip-on smart textile patch will be used as a databank and a communication device, which can automatically collect the health related information and transmit these information to the proper physician in real-time. Uniforms for firefighters, security guards, and special mission personnel will provide all required information on their health and safety condition, as well as transmit the information remotely to a central command office, as one can see, the possibilities are endless. "Smart and intelligent" structures are defined as structures capable of monitoring their own "health" condition and structural behavior, such structures are capable of sensing external environmental conditions, making decisions, and sending the information to other locations. They consist of special types of sensors, signal processing electronics, data acquisition, actuators, control devices, and a communication network embedded into the structures. The conventional sensors, actuators, electronics and networking lines currently available, are not technologically mature for such applications. New classes of miniature devices and networking systems are urgently 
needed for such applications. Furthermore, methodology for integration into materials/structures needs to be developed. Fiber optic technologies are compatible with this type of application and present the best choice for the time being.

The development of fiber optic sensor technology, whose overall geometrical dimensions are miniature, has opened the possibility of embedding fiber optic sensing elements within various structures. This allows for in situ monitoring the state of the material, in real-time. These sensing elements can be embedded with minimal interference to the structure, and can be used as cradle-to-grave sensors.

To this end, in response to the challenges inherent in the development of smart/intelligent structures, a highly qualified multidisciplinary team was organized in the middle 1990s at Drexel University, in collaboration with Photonics Laboratories, Inc. This multidisciplinary team was set on focusing on the development and application of smart structures in industrial, military, and biomedical engineering. The team's work has focused on the design and development of miniature fiber optic sensors and on the integration of these sensors, as well as communication links of optical fibers and electrical wires, into various composites and textile based materials. Integrating lightweight miniature sensors and networks into smart structures will provide tremendous advantages for many applications, forming the basis for the development of intelligent biomedical systems for health monitoring, diagnostics, and treatment.

In this paper, two examples of the successful work achieved so far in biomedical applications will be presented and discussed. The first one is based on the development of a smart bone fixation device for rehabilitation and treatment. This device includes a smart composite bar that can sense physical stress applied to the fractured bones, and send the information to the patient's physician remotely, during the healing process. The second is on the development of smart fabrics for many applications including health monitoring and diagnostics. Successful development of such smart fabrics with embedded fiber optic sensors and networks is mainly dependent on the development of the proper miniature sensor technology, and on the integration of these sensors into textile structures. Before presenting these two examples on smart structures however, it is a prerequisite to discuss the miniature sensor technology that has been developed for these applications.

\section{FIBER OPTIC SENSOR TECHNOLOGY}

Current research in fiber optic sensors relates perturbations, to changes in the total intensity of the light signal transmitted through the optical fiber. Other methods involve launching coherent polarized light through an embedded optical fiber and observing the changes in the polarization or phase of the launched signal with the applied external perturbation.
Sensors based on intensity modulation are simple and cost effective. However, they provide limited sensitivity. Phase and polarization modulation type sensors provide much better sensitivity, but they are bulky and require laser sources and expensive techniques for detection. To overcome these limitations, a novel technique, based on monitoring the modal power distribution (MPD) in multimode fibers, was developed. This technique provides a sensitive and inexpensive methodology for miniature sensors applications (El-Sherif 1989; El-Sherif 2001).

The principle of operation of the developed technique is based on spatial intensity modulation (SIM) of the modal power in multimode fibers. Within a multimode optical fiber, optical signals propagate according to the modal structure of the fiber and the boundary conditions. Altering the boundary conditions of an optical fiber induces modal coupling and results in MPD modulation. The coupledmode-theory can be employed for the analysis of the MPD modulation (EL-Sherif et al 2000; El-Sherif et al 2001). The MPD within a multimode fiber is a function of the geometry (size) and the optical properties (core and cladding indices) of the fiber and the light launching conditions. Deforming the fiber by mechanical stresses or other forms of perturbation results in modal power modulation, which can be exploited in sensing the applied signals. The measurements of the distribution and the subsequent redistribution of the modal power can be accomplished by scanning the far-field pattern at the fiber end using a charged couple device camera (CCD camera), or by using one or more photodetectors positioned at a specific location in the far-field zone, as shown in Figure 1. The modal launcher is a single or array of light emitting diodes (LEDs), used to excite a limited group of modes within the optical fiber, and the modal analyzer is the detection system of the modal power positioned at the output end of the optical fiber, in the farfield zone. As an example, a step index silica fiber of $100 \mu \mathrm{m}$ diameter was excited at 10 degrees off-axis. The 2-D farfield pattern (MPD) and intensity profile were scanned and recorded by a CCD camera as shown in Figure 2a. When the fiber was under stress, the recorded far-field pattern shows inter-modal coupling and redistribution of the modal power (see Figure 2b). As the applied stress was increased, considerable rearrangement of the modal power was recorded in a similar way. These two figures indicate that continuous variation of the applied perturbation will result in a respective change in the MPD, in a very sensitive manner. For a cost effective and miniature sensor configuration, the CCD camera can be replaced by a single or number of photodetectors located at key positions in the far-field. These detectors can be coupled to simple biasing and signal processing circuits to indicate the spatial variation in the optical signal. Using a light emitting diode as the optical source and a regular photodiode for detection will provide a miniature and inexpensive technique compared to the phase or polarization modulation techniques.

The sensitivity and the dynamic range of this type of embedded sensors are related to the modal structure of 


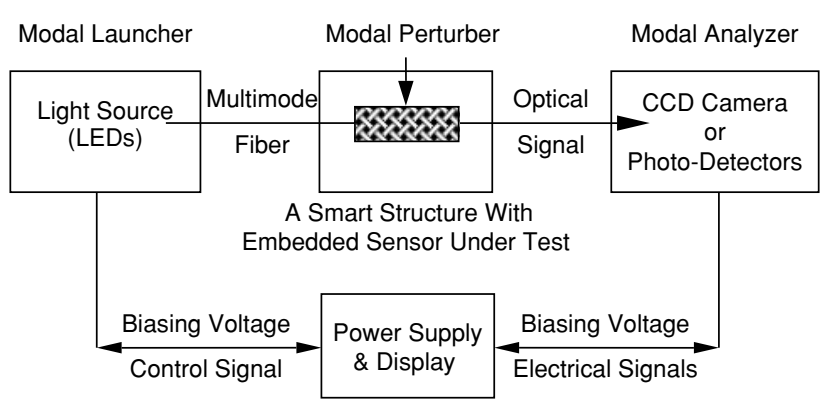

Figure 1 The general block diagram of the developed characterization method.

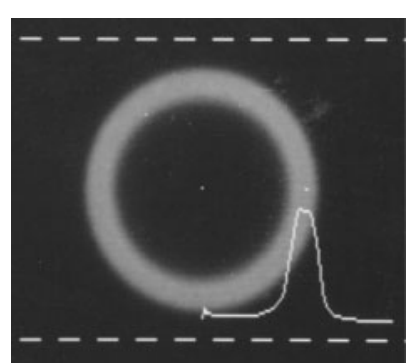

(a)

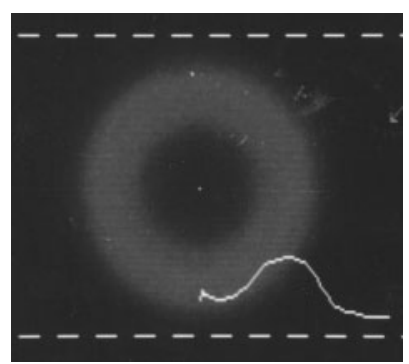

(b)
Figure 2 The 2-D image and the horizontal intensity profile of the far-field pattern measured at the center before (a) and after (b) the presence of perturbation.

the fiber, and to the mechanical behavior of the materials surrounding it. In application to composite structures, the concentration of strain induced in the optical fiber influences the level of sensitivity, which is strongly dependent on the modulus of elasticity of the surrounding materials, such as coating and resin. A properly coated optical fiber can offer better performance over that of the bare fiber.

In conclusion, a compatible method suitable for smart structures with embedded fiber optic sensors has been developed. Experimental and theoretical feasibility studies show that the developed sensing technique is sensitive, inexpensive, and can be manufactured in microstructure components.

\section{SMART BONE FIXATION DEVICE}

It is quite common for patients to undergo orthopedic surgical procedures to correct deformities and/or realign fractured bones of the lower extremities. This can involve any fracture from the foot to the hip joint. Depending on the physical abilities of the patient involved, the rehabilitation potential for patients will be compromised if they are putting too much pressure on the area that has been injured. At the present time, there is no system capable of self-monitoring of patients as they begin to bear weight on that extremity, rather, reliance is placed on verbal communication between the patient and a physical therapist. The therapist must monitor the patient day after day in a rehabilitation center to be sure that the patient is not

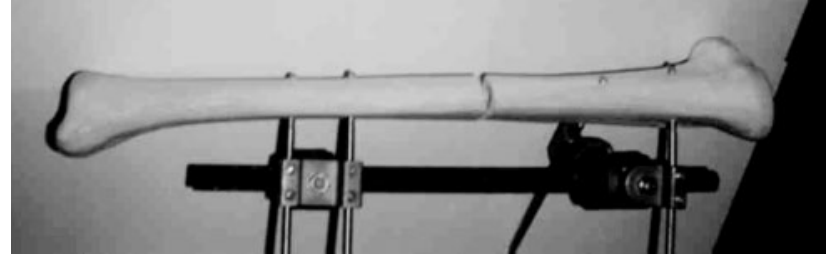

Figure 3 Bone fixation devices constructed of a hex-bar fixed in the bone parts using four long screw bolts.

putting too much weight on the extremity. If the therapist frightens the patient by threatening them with predictions of dire consequences from over-utilizing their legs and putting too much weight on the involved area, the patient will probably respond by not putting any pressure on the leg at all. Therefore, it would be very helpful for the patient to have a mechanism that give them feedback in their rehabilitation program, with the therapist monitoring the compliance that the patients show in the program. If the therapist's verbal instructions are understood, patients can be released to their own devices much sooner. If the therapist does not believe that the patient understands the instructions that have been given, this device would reinform the patient at all times, confirming the therapists initial impression and prevent re-injury.

Available technology depends on the use of strain gauge sensors, which are unreliable or incompatible in most applications. Therefore, it would be helpful to have a better technology for sensors applied to rehabilitation systems, which would measure the amount of pressure being exerted over a specific point or several points. If a reliable method to measure localized pressure were to be available, the surgeon could develop information that would allow for postoperative rehabilitation programs for patients, specifically based and modified according to the data received from these devices. Therefore, a post-operative rehabilitation program could be formulated on a scientific basis rather than by trial and error.

Therefore, the goal of the R\&D program conducted on a bone fixation device is to develop a device that can contribute to the quality, efficiency, and effectiveness of the patient rehabilitation process, as well as to the reduction of health care costs. Specifically, the objective is to develop an optical fiber remote sensor embedded within orthopedic bone fixation devices to improve patient rehabilitation following orthopedic injuries. The work plan has been successfully completed, and a demonstration prototype of the sensor system has been developed and tested. The sensor operates on the principle of MPD modulation explained before for multimode optical fibers application.

The Smith \& Nephew's bone fixation device was selected for demonstration purposes. The device is shown in Figure 3. The device consists mainly of a 12 in. long composite hex-bar, as shown in Figure 4. The bar is used to hold the broken bones together, by using four long screw bolts, as shown in Figure 3. 


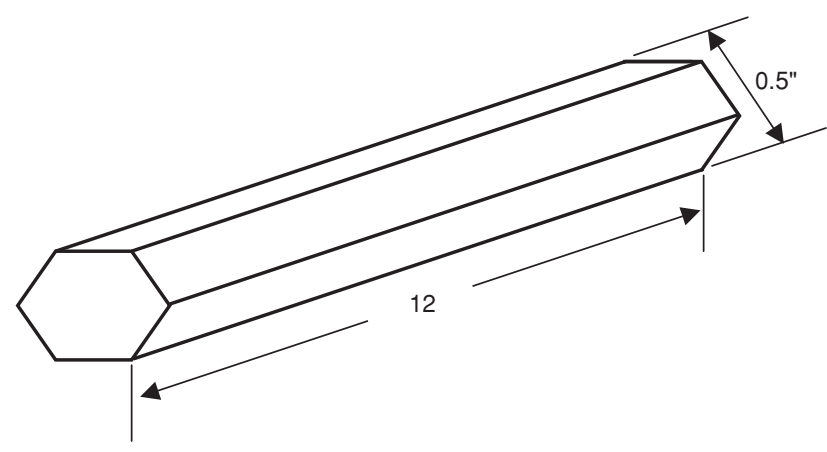

Figure 4 Hex-bar geometry.

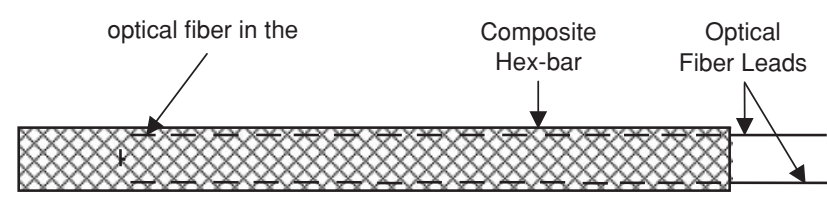

Figure 5 The optical fiber is placed between the first and second outer layers of the fiber network.

The primary objective is to develop a fiber optic pressure sensor for monitoring patient rehabilitation. A multimode optical fiber was embedded in the 12 in. composite hexbar used in the Smith \& Nephew's bone fixation device, as shown in Figure 5. The embedded fiber is used as the sensing element of the fiber optic sensor. The sensory system includes: the sensing fiber, LED, optical coupling devices, opto-electronic circuits, remote $\mathrm{RF}$ transmitter/receiver, and a digital liquid crystal display (LCD) for output indication. The remote $\mathrm{RF}$ transmitter/receiver is used to provide remote control for monitoring physical pressures applied to the fractured bones during their healing process. The R\&D work includes design, fabrication, and testing. The opto-mechanical behavior of the sensing element depends on the characteristic response of the embedded fiber to external loads. The system concept of monitoring external pressure applied to the bone fixation device has been proven experimentally. The remote sensor demonstrates the idea of monitoring pressures applied to the bone fixation device. It is anticipated that such a system can be utilized for post-injury therapy and for diagnostic studies of postoperative patients during their rehabilitation.

\section{Design and fabrication of a smart hex-bar}

The hex-bar is made of reinforced composites consisting of a multi-layer 2-D braided fiber network. The fiber network is made of Hercules AS-4 carbon fibers. The design components include preform, mold, and sensor design. The preform was braided on a 24 carrier 2-D braiding machine. The dimensions of the hex-bar are $0.5 \mathrm{in}$. diameter and 12 in. in length, as shown in Figure 4. Since the preform has a multi-layered structure, the optical fiber was embedded under the outside layer, and was exposed at the end of the hex-bar, as shown in Figure 5.
The preform was consolidated with polyester fiberglass resin in a special mold. The resin was mixed first with the required hardener. The ratio of hardener to resin is 14 drops per ounce of resin. With this ratio, the resin gels in about $10 \mathrm{~min}$ and is completely cured in $2 \mathrm{~h}$. Before being put into the mold, the resin is applied to the preform using the wet lay-up method.

\section{Fiber optic sensor}

The concept of sensing the stresses applied to the hexbar is based on the MPD technique explained before. If only higher-order modes are excited in a fiber, the far-field pattern received at the fiber end will be a clear circle with a dark area in the center. The incidence angle of the light has to be adjusted in such a way that only higher order modes are excited in the optical fiber. Mechanical stresses applied to the hex-bar will cause a redistribution of light intensity in the far-field pattern. As a result, the light intensity at the center of the far-field pattern will change. Such detection of these changes can be used to sense the stress applied to the hex-bar.

A typical red LED is used as a light source, and a photo detector is used to detect the changes of the far-field pattern. The end surfaces of the optical fiber are finely polished, and the light incidence angle is adjusted such that, a light ring is formed in the far-field of the fiber. The output light of the fiber is coupled to the photodetector using a holder designed specially for this application. The position of the photodetector, localized in the far-field at the end of the fiber, is critical to maximize the sensitivity of the sensor. After fine adjustments are made, the fiber, LED and the detector are all fixed into position using optical glue.

The sensor design consists of two main parts, the transmitting part and the receiving part. The transmitting part consists of a light source LED, power supply, switch, voltage control circuit, and an optical coupling device to couple the optical signal to the optical fiber. The receiving part consists of a photodetector, a current to voltage transfer circuit, an amplifier, and power supply. The transmitting part is connected to one end of the fiber, and the receiving part of the circuit is connected to the other end of the fiber.

\section{RF transmitter/receiver}

An RF transmitter/receiver is designed and developed for remote sensor applications, for real-time monitoring of the physical pressures applied to the bone fixation device. The frequency and power of the RF signals are selected based on the safety regulations required for this type of devices. The maximum distance between the transmitter and the receiver is designed at $25 \mathrm{~m}$ and can be increased up by $50 \mathrm{~m}$. The main function of the transmitter is to convert the dc output of the photodetector to a low frequency signal using a voltage to frequency converter. A buffering circuit is used between the voltage to frequency converter 


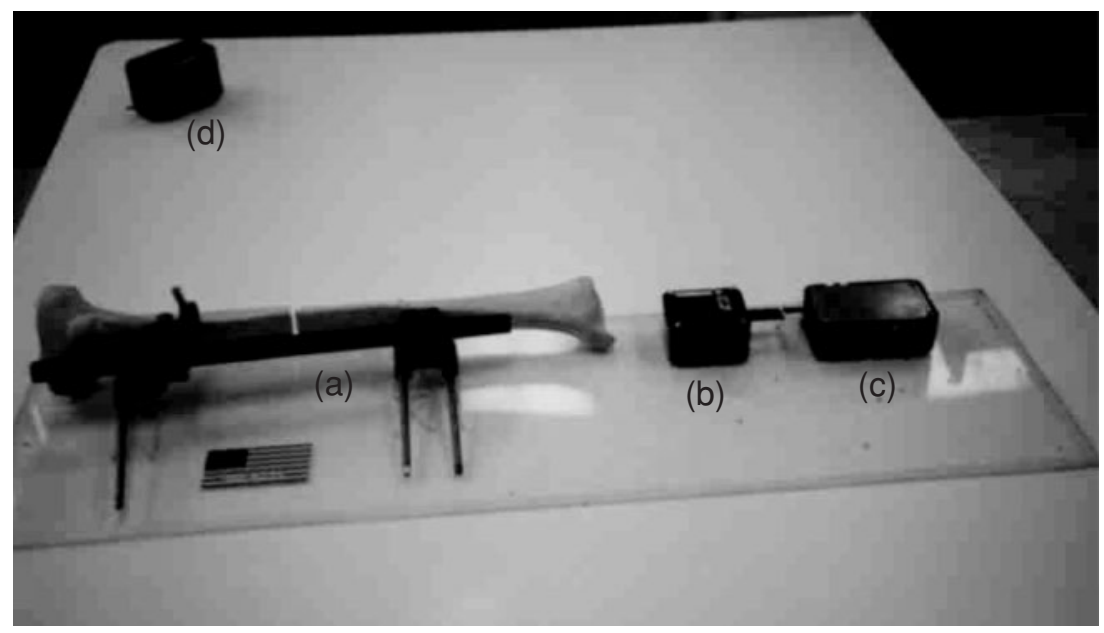

Figure 6 The developed smart bone fixation device and the remote sensing system, where (a) is the smart bone fixation device, (b) is the optoelectronic circuit, (c) is the wireless RF transmitter, and (d) is the wireless RF receiver and indicator.

and the RF transmitter. The transmitter generates the carrier frequency. This signal is transmitted through a wire antenna. At the receiving end the signals are detected by a loop antenna and amplified before they are converted to variable voltage signals using a frequency to voltage converter. Then, the signals are fed to an LCD for reading the sensor output. A smart bone fixation device was designed, manufactured and successfully tested for remote operation. The developed system is shown in Figure $6 \mathrm{a}$. It consists of four main components; (1) the smart bone fixation device; (2) the opto-electronic components for the sensor; (3) the RF transmitter unit; and (4) the RF receiver unit, as shown in Figure $6 \mathrm{~b}$. Note that in Figure $6 \mathrm{c}$, the RF receiver is located, far from the other three components, for remote controlled detection of the information. The developed system demonstrates the idea of using remote fiber optic sensors with rehabilitation equipment, for realtime measurements and continuous monitoring of patient health conditions.

\section{Results and Discussions}

The developed sensory system measures the instantaneous pressure applied to the bone fixation device. The output is shown on the liquid crystal display, as shown in Figure $6 \mathrm{~d}$ (the wireless RF receiver and indicator). This type of sensors requires calibration after manufacturing. Also, the sensor sensitivity and dynamic range would be tailored during the designed and the manufacturing process to match the application standards, i.e. if it is for heavy or lightweight persons. The sensitivity can range from a few grams to $100 \mathrm{gm}$ and the dynamic range can be from a few $\mathrm{kg}$ to over a $100 \mathrm{~kg}$. This tuning can be achieved by pre-coating of the embedded optical fiber, as explained before, and by embedding the fiber at different distances from the surface of the hex-bar. The sensitivity of the developed demonstration system was $25 \mathrm{gm}$ and the dynamic range was about $32 \mathrm{~kg}$.

\section{SMART SHIRT FOR HEALTH MONITORING AND DIAGNOSTICS}

\section{General}

In accordance with the advancement of the health monitoring and diagnostic systems, this section presents a novel approach on the development of a smart shirt for health monitoring and diagnostics applications. Three major $R \& D$ programs were conducted, in collaboration with the Department of Defense, on the development of smart textile/fabrics for various applications, which can be transformed for health monitoring and diagnostics. The first program was on the development of the methodology for integration of optical fibers and electronic wire into textile fabrics during the manufacturing process, using commercially available textile machines (El-Sherif and Yuan 1999). The optical fibers and the electric wires will be used for building sensors and devices within the textile fabrics, and for transmission of the information. The developed methodology can be used for processing and manufacturing different types of the smart shirt, based on the textile fabric structures and materials. The second program was on the development of Smart Soldier's Uniforms with embedded fiber optic chemical/biological sensors for early warning on existence of toxic or biological substances in the space surrounding the soldier (El-Sherif et al 2000). This can be modified to analyze the patient's breath and check for the quality of the environmental conditions, such as oxygen level. The third program was on the development of smart parachute integrating fiber optic stain/stress sensors for measuring actual stresses and vibrations induced in the parachute canopy during the airdrop and the inflation of the canopy (Marcuse 1973). The smart parachute includes electronics and an RF transmitter/receiver for signals processing, multiplexing and transmission of the sensors output to a ground station (Radhakrishnan and El-sherif 1996). This type of smart fabrics can be transformed to checking heartbeat measurement or monitoring 


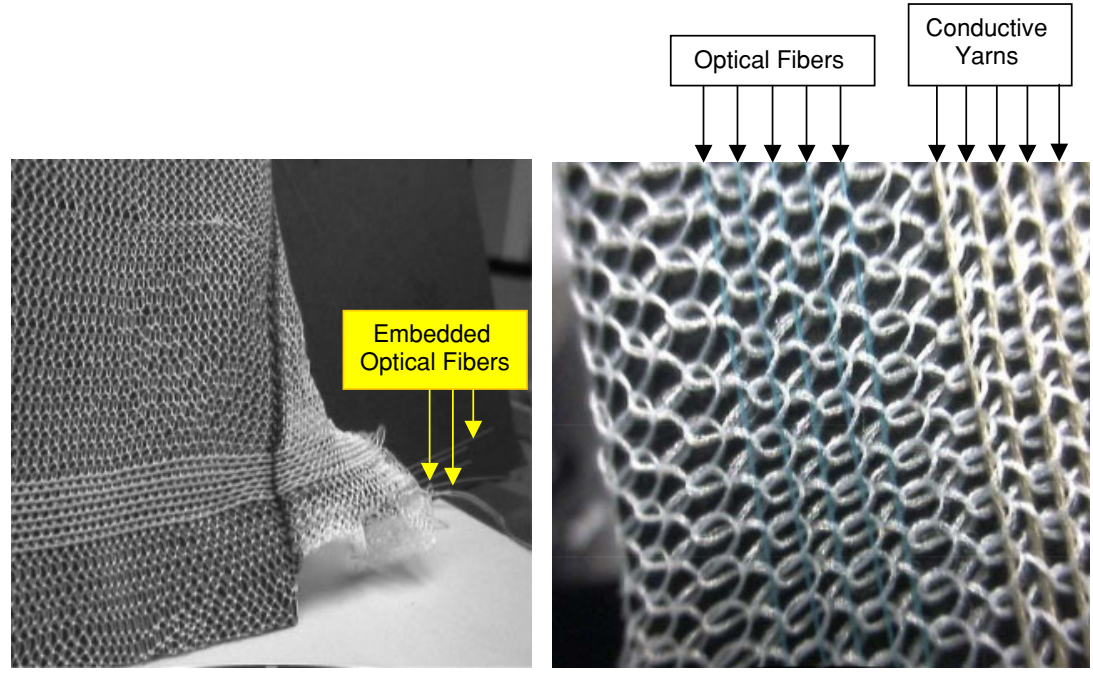

Figure 7 Knitted textile fabric with embedded optical fibers and conductive yarns (left), and the macroscopic picture of the knitted textile structure (right).

other similar mechanical motion or vibration, such as chest motion under various breath conditions. The developed remote sensor system can be used to transmit the information instantaneously to the patient physician.

The technology developed through these three programs will be used in the near future for the design and manufacturing of the Universal Smart Shirt, for health monitoring and diagnostics. The developed sensors are highly sensitive, and designed in microstructures to be compatible with the textile applications. In this paper, samples of the results achieved on integration of optical fibers and electric wires into textile structures during the regular manufacturing process will be presented. Also, results on the application of these integrated sensors as a strain and vibration measurement will be presented and discussed.

\section{Integration of optical fibers and wires into textiles}

The development of textile fabrics with embedded optical fibers and electric wires requires a full understanding of textile structural behavior and geometry. For proper integration of optical fibers, the processing parameters and final shape conditions of the textile structures should be carefully identified. Three types of basic textile structures (knitted, woven and nonwoven) were under consideration for development as smart textiles.

\section{Knitted structures}

There are two main knitted structure types, warp knitted and weft knitted. The yarns in a knitted textile material are in the form of inter-loops that are subjected to very tight bends at a very small radius of curvature. The idea of replacing one of the yarns with optical fiber is not acceptable for these structures. The tight bends may cause extreme losses in optical signals and may even result in their mechanical failure. Therefore, for knitted structures, optical fibers cannot be inserted in the same way that textile yarns are interwined, however, optical fibers and electric
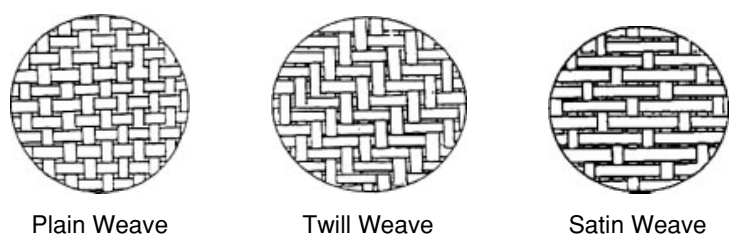

Figure 8 Three forms of textile weaving structures: (a) plain weave, (b) twill weave, (c) satin weave.

wires can be integrated in a straight line, interlacing with the loops, as shown in Figure 7. In this case the integrated optical fibers and wires can have an acceptable path in the textile material without critical bending and mechanical deformation.

\section{Woven structures}

In woven textiles, there are three forms of woven structures: plain, twill, and satin. The yarns are interlaced and subjected to various bending conditions as shown in Figure 8 . The twill weave has a less bending curvature than plain or satin structures. Therefore, the best integration of optical fibers is in the twill weave structures, where the fiber is subjected to the least bending in curvature. In general, in woven structures, the optical fiber can be integrated during the regular manufacturing process by replacing one of the textile yarns with the optical fiber, as shown in Figure 9, for a plain woven structure.

\section{Non-woven structures}

Non-woven textiles are made up of sheet materials composed of more or less randomly oriented fiber segments bonded together. There are basically two structural features in non-woven structures: the mode of web formation and the mode of bonding. The most common web formation consists of layers of short fibers laid on top of each other. There are three main types of bonding: entanglement, sticking the fibers together, and stitching through 


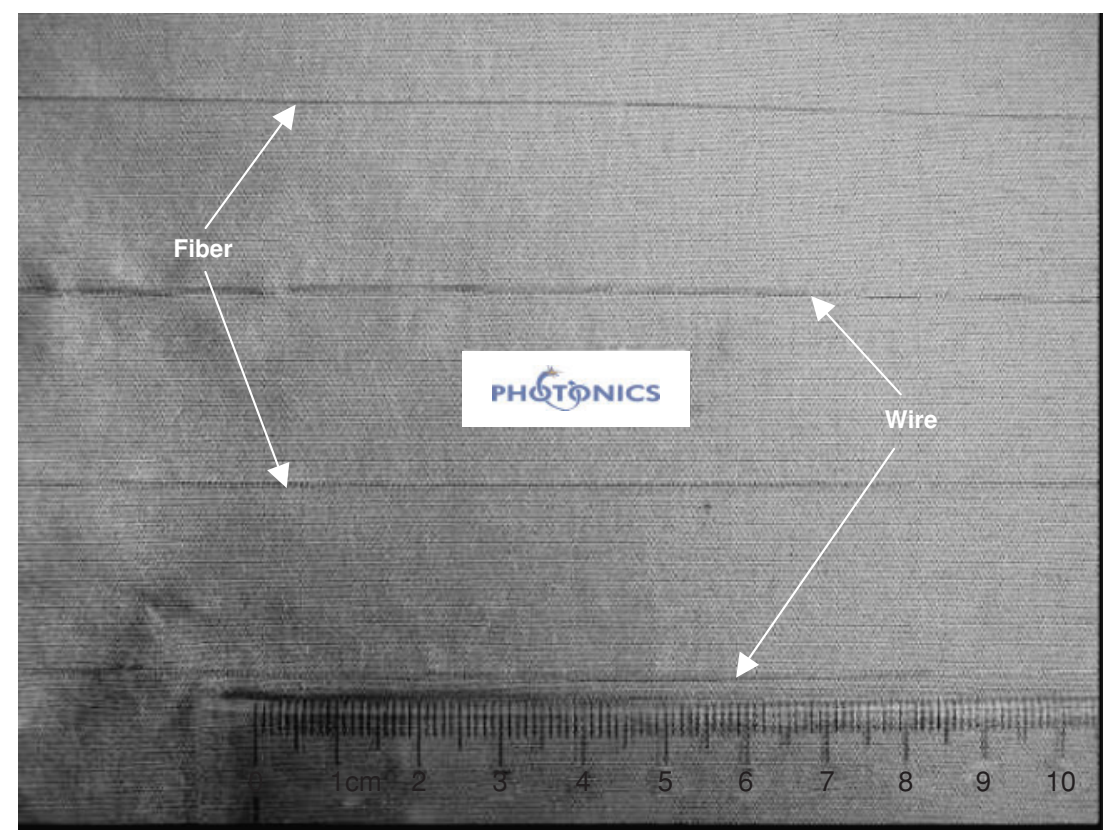

Figure 9 Different types of optical fibers and electronic wires integrated into plain woven structure.

the fabric. Thus, the only way for optical fibers and electric wires to be integrated into non-woven structures is by placing them between the sheets (or layers). The fibers/wires orientation can be in a straight line or with minimum bending curvature. Therefore, the integration of optical fibers in non-woven structures is much easier than in knitted or woven structures.

\section{Smart textiles with embedded fiber optic sensors}

The successful integration of optical fibers and fine electric wires into textile structures has led to the development of a number of applications. One of these applications is the smart parachute, which has the ability to predict the opening forces and to measure the deformation of the parachute canopy fabric during airdrop. Smart parachutes with embedded fiber optic sensors can be used for real-time characterization of the dynamic structural behavior of parachutes during airdrop. Another developed application of smart textiles is the smart uniform, which has the ability to sense environmental conditions. The detection of biological or toxic substances is based on the concept of modifying optical fibers (passive conductors) to be chemically sensitive fibers. This modification is achieved by replacing the passive cladding material in a small section of the optical fiber with a chemically sensitive agent (Snyder and Love 1983). When these sensors are incorporated into clothing, such as uniforms for fire fighters, security guards, and special mission personnel, they would provide instantaneous early warning of the presence of chemicals or toxins in the ambient environment. A large number of technical reports and papers were published, recording the progress achieved so far on these two applications (Yuan and El-Sherif 2003). These applications will form the basis for the development of the Universal Smart Shirt, for health monitoring and diagnostics. This shirt can also be used to monitor heart conditions, blood analysis and circulation, injury conditions, and other health issues, using the principles developed through the smart parachutes and the smart uniforms. In situ measurements of perturbations induced in textile fabrics, will be applied for sensing heartbeat, breath conditions, and others. The application of smart textiles in self-monitoring of induced stresses and vibrations within the structure was successfully conducted, using sensitive, simple, and inexpensive methods. Samples of the developed methodologies will be presented next.

\section{Monitoring of induced stresses in textiles}

Two types of fiber optic sensors were developed and integrated into nylon fabrics for sensing quasi-static and dynamic loads. One of these types is based on the fiber Bragg grating (FBG) sensors, and the second type of the sensors is based on the MPD sensor, explained before (Radhakrishnan and El-sherif 1996). This MPD sensor type has the advantages of being inexpensive, highly sensitive, flexible, and very small, which are the requirements for application to the medical smart shirt. Since the application of FBG sensors for strain measurement is well known, it was used in parallel with the MPD sensors for calibration, and back check on the MPD results. The application of the MPD sensors embedded in smart textiles is discussed next.

Experimental work was carried out for the validation of the MPD technique in measuring quasi-static and dynamic strains in textile structures. Quasi-static and dynamic tests were performed on processed samples. Strands of optical fibers are embedded into small pieces of woven nylon fabrics and tested. The quasi-static test results are used to build the relationship between the optical signal and the 


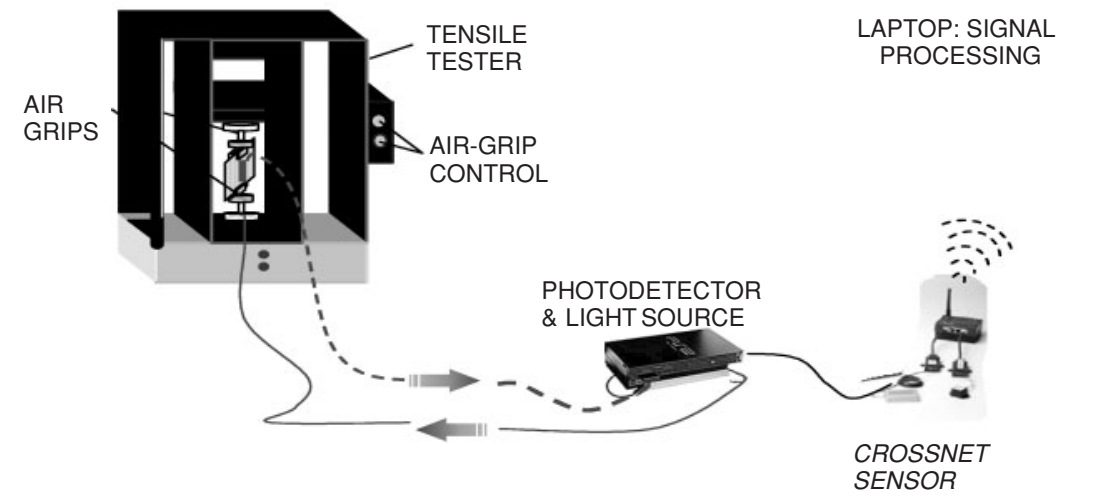

Figure 10 Smart textile with embedded MPD sensor, under quasi-static test, with remote control system.
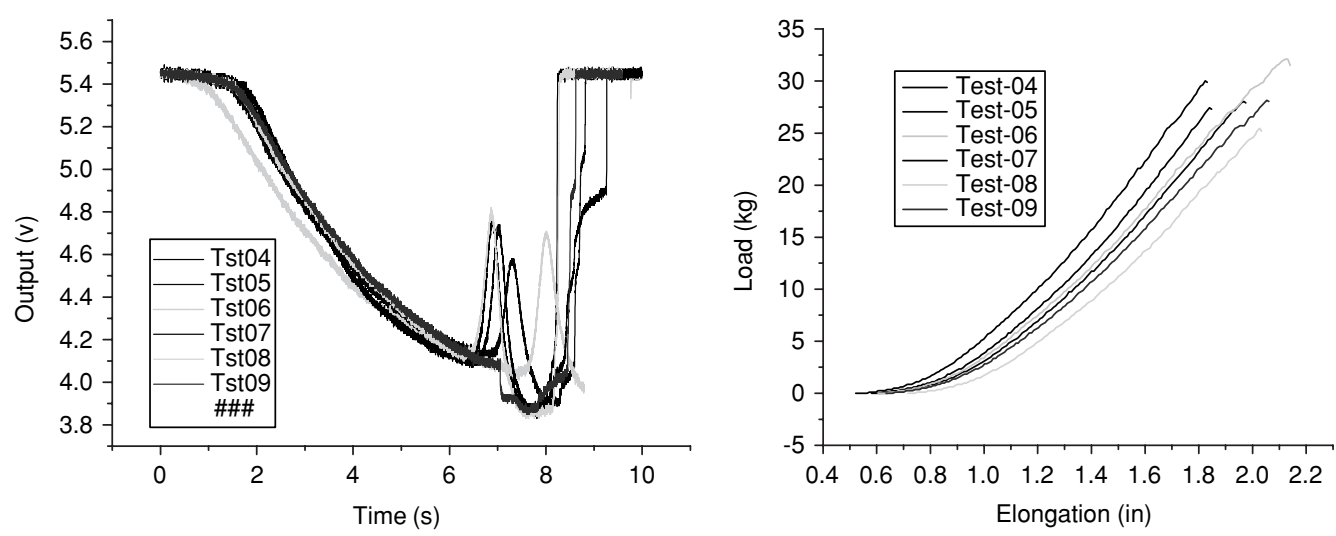

Figure 11 Optical sensors output (left) and the tensile tester outputs (right) for the six tested samples.

mechanical behavior of the fabric and thus to predict the external perturbation on the smart fabric during dynamic application.

The experimental set-up for the remote sensor application is shown in Figure 10, where the MPD sensor output is transmitted remotely to another location, using the developed Crossnet RF transmitter/receiver. By using a photodetector positioned at a certain location in the farfield pattern, at the fiber end, information on the modal power redistribution can be continuously recorded. The tensile tester will give the relation between the loads and the elongation of the fabric structure.

The quasi-static tests were carried out in a uniform, systematic way. Each sample is mounted on the tensile tester, and the optical fiber is connected from one end to a light source and from the other end to a photodetector and a RF transmitter. The tensile tester pulled the fabric a certain amount and then the output data was recorded for the load, elongation, and the sensor output. This process was continued until the fabric started to tear. Figure 11 shows the sensor output (volt vs. time) and the tensile tester output (load vs. elongation) for six tested samples.
It can be seen that the sensor output was in full agreement with the tensile tester output for the first $6 \mathrm{~s}$, before the fabrics started to tear.

For dynamic tests, a test set-up was designed to study the dynamic behavior of the smart textiles. A free falling drop test set-up was designed, as shown in Figure 12. Several tests were carried out using different weights and different dropping distances. In each case, the output of the photo-detector was recorded. Figure 13 shows the photodetector output, voltage versus time, for three samples tested at $6 \mathrm{lb}$ weight and $1 \mathrm{ft}$. drop height. The sensor output for the three samples is identical. The first pulse records the drop test output data, and the following pulses represent the oscillation information. The tests were repeated for different weights and heights.

The major observation to be deduced from the collected data is that the height of the first pulse and the number of major pulses increases as the dropping distance increases. A second observation is that the delay time between the first two major pulses increases as the dropping distance increases. This increase in delay time reflects an increase in the time due to the load motion to bounce back 


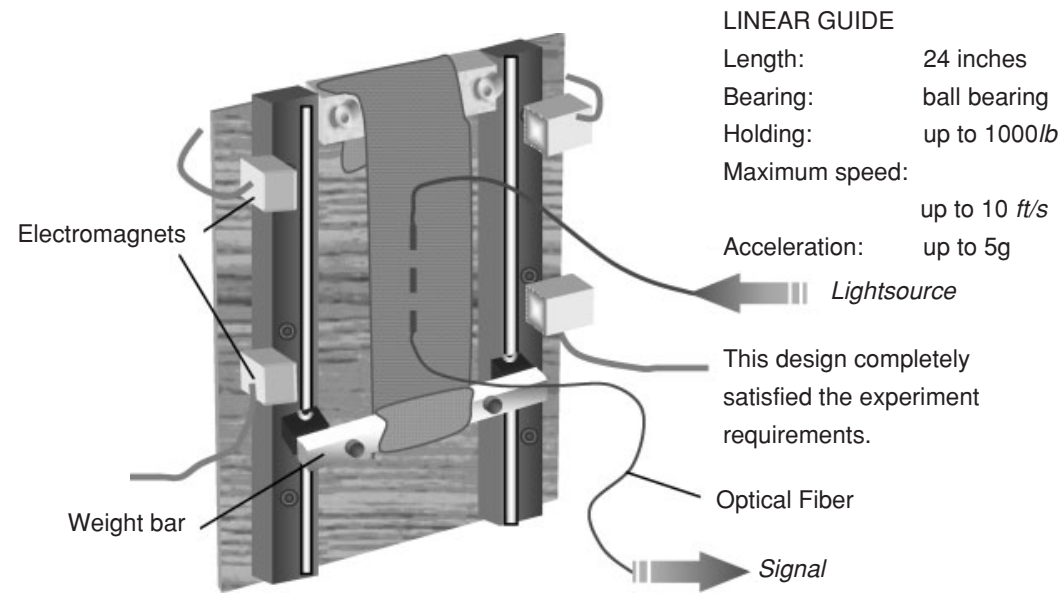

Figure 12 Experimental set-up designed for free-falling drop tests.

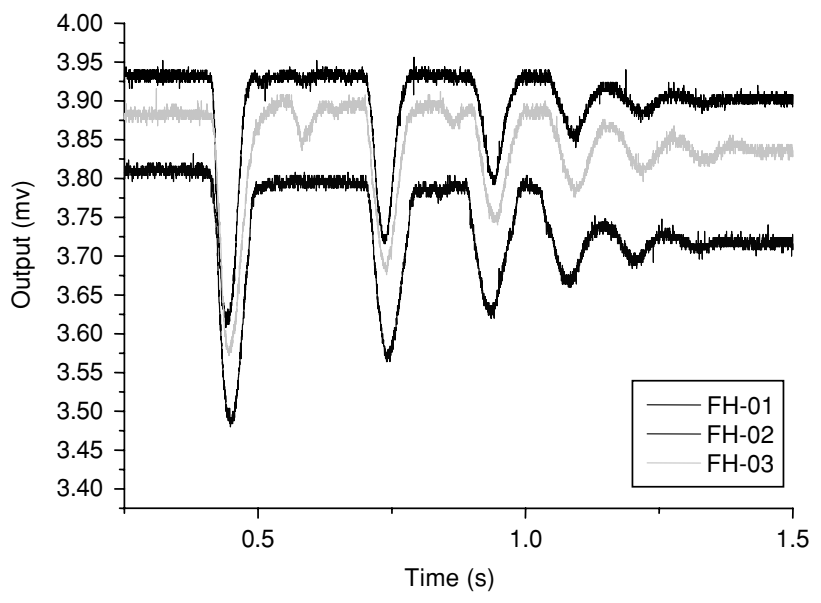

Figure 13 Drop test results of MPD sensors for three samples.

for the second cycle of oscillation. As a result of these drop tests, a clear relationship exists between the dropping weight and dropping distance on one side and the pulse widths, time delay between the first and second pulses and the number of pulses on the other side. The number of experiments conducted was enough to draw a general conclusion on these relations, that the developed methodology can be applied for measuring vibrations induced in textile fabrics. In the smart shirt application, the recorded data can be directly translated to determine the heartbeat conditions or the breath conditions in infants, or other health related conditions. Based on the successful results achieved in the laboratory tests (quasi-static and dynamic) and the mechanical analysis and modeling, a correlation function between the induced strain and the sensors output has been developed.

\section{CONCLUSION}

In conclusion, the idea of using smart structures in biomedical applications will have a strong impact on the advancement of health monitoring and diagnostics systems. The smart bone fixation device presented in this paper is an example of how smart structures can be integrated into various rehabilitation devices and artificial body parts for continuous health monitoring and improvement. Also, the developed remote sensory system, embedded into woven fabrics, has been proven to operate successfully in monitoring any perturbation induced in the textile fabrics, as well as monitoring environmental conditions. Furthermore, the methods developed for the manufacturing of the smart parachute and the smart uniform can be applied to the development of the smart shirt. In addition, the developed wireless remote sensor capability can be applied to the smart shirts for continuous health monitoring by the patient physician from a remote location. Finally, the value of the MPD technique in smart structures applications, where the requirement of miniature structures and sensitivity are of great importance, has been successfully demonstrated through these smart textiles and smart composites applications. To this end the potential application of smart structures in biomedical industry/technology is limitless.

\section{ACKNOWLEDGMENT}

The author would like to acknowledge the U.S. Army Research Office, NC, and the U.S. Army Soldier and Biological Chemical Command, Soldier System Center, Natick, MA, for their financial support and technical collaboration through the MURI-ARO project No. DAAH04-961-0018, the BAA project No. DAAD16-01-C-0054, and the SBIR phase II project No. DAAD16-01-C-0003. Also, the author would like to thank the members of the Fiber Optics and Photonics Manufacturing Engineering Center, at Drexel University, and the technical staff at Photonics Laboratories Inc., for their valuable technical contribution to the reported research. 


\section{REFERENCES}

El-Sherif MA. 1989. On-fiber sensor and modulator. IEEE Trans Instrum Meas, 38(2):595-598.

El-Sherif MA. 2001. The Final Technical Report on Sensors and Smart Fabrics. The MURI-ARO (Army Research Office) project on Functionally Tailored Textiles. Contract \#DAAH 01-96-1-0018.

EL-Sherif MA, Fidanboylu K, El-Sherif D et al. 2000. A novel fiber optic system for measuring the dynamic structural behavior of parachutes. F Intell Mater Syst Struct, 11:351-359.

El-Sherif MA, Li M, El-Sherif D et al. 2001. Fiber optic system for measuring the structural behavior of parachute airdrop: Quasi static and dynamic testing. Chang FK. Structural health monitoring. Boca Raton, FL: CRC. p. 733-741.
El-Sherif MA, Yuan J. 1999. Fiber optic sensors and smart fabrics. The Fourth ARO (U.S. Army Research Office) Workshop on Smart Structures. Pennsylvania.

El-Sherif MA, Yuan J, MacDiarmid A. 2000. Fiber optic sensors and smart fabrics. F Intell Mater Syst Struct, 11:407-414.

Marcuse D. 1973. Coupled mode theory of round optical fibers. $B S T \mathcal{A}, 52: 817-842$.

Radhakrishnan J, El-Sherif MA. 1996. Analysis on spatial intensity modulation for fiber-optic sensor applications. Opt Fiber Technol, 2:114-126.

Snyder AW, Love JD. 1983. Optical Waveguide Theory. New York: Chapman and Hall.

Yuan J, El-Sherif MA. 2003. Fiber optic chemical sensor using polyaniline as modified cladding material. IEEE Sensors $\mathcal{F}$, 3:5-12. 

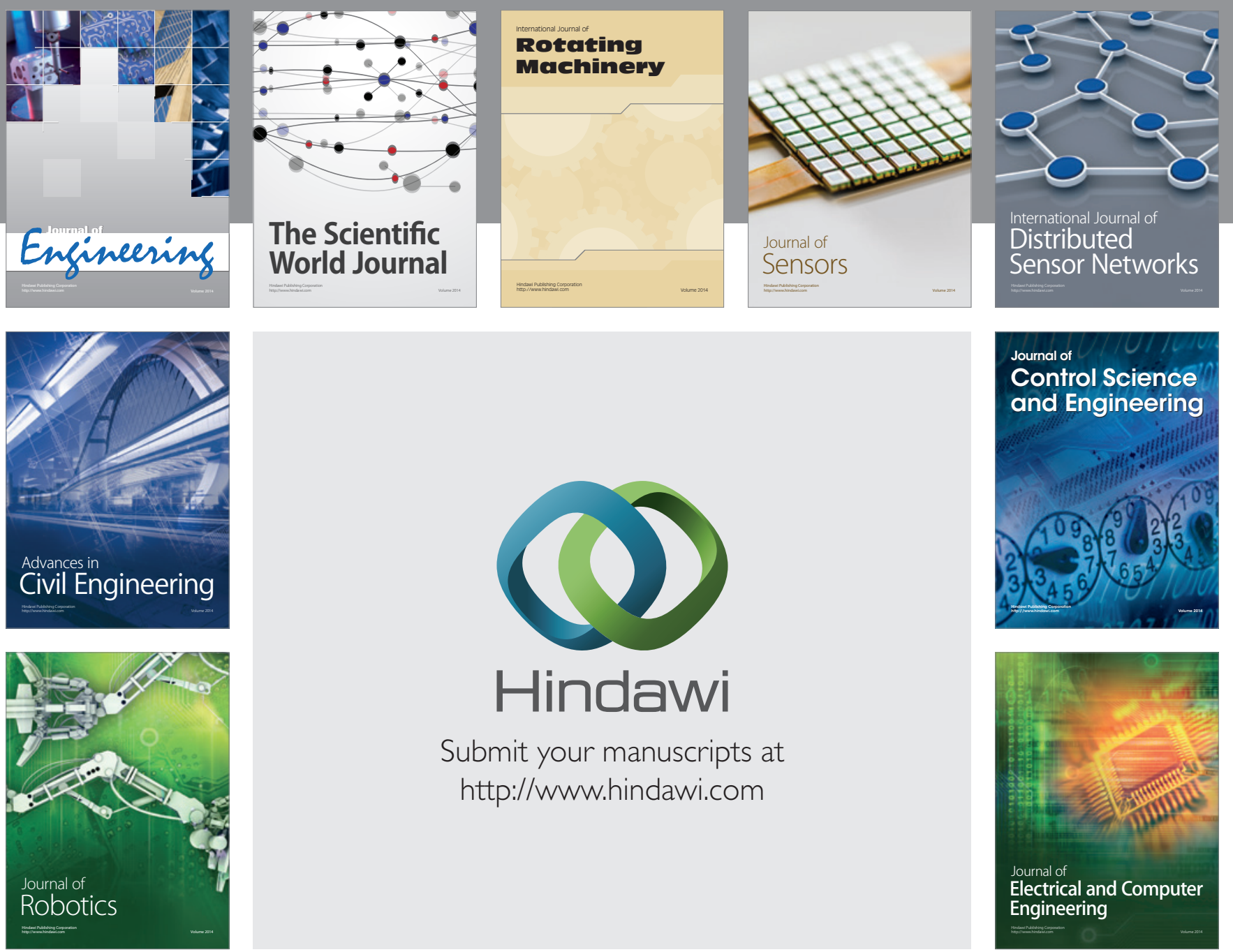

Submit your manuscripts at

http://www.hindawi.com
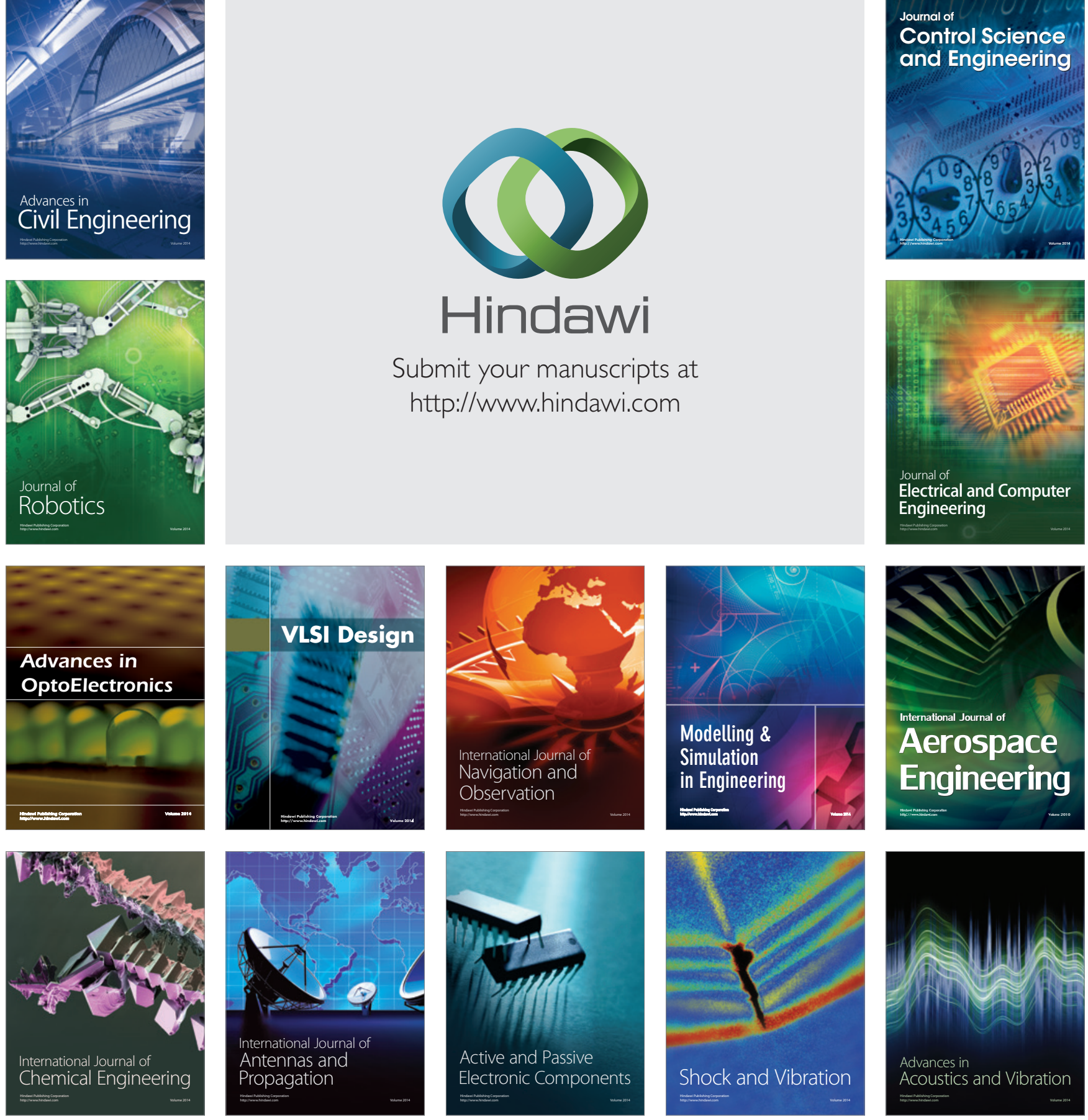